\title{
THE PREPARATION OF OPTICALLY STABLE SUGAR SOLUTIONS FOR COLORIMETRIC ANALYSIS ${ }^{1}$
}

\author{
By J. F. Brewster and F. P. Phelps
}

\section{ABSTRACT}

The successful application of colorimetry in the sugar industry is dependent upon the development of a satisfactory technique for preparing sugar solutions having a satisfactory degree of transparency. In the present paper existing methods have been revised. The process of clarification has been shortened and the transparency of the solutions improved. These results have been accomplished by increasing the concentration of the dry substance or total solids, dissolving and filtering the sugar products hot, and developing the application of better filters. In addition, other details of manipulation have been improved.

\section{CONTENTS}

I. Introduction ... 365

II. Filters. 366

III. Calculation of weights

IV. Preparation and filtration of the sample 367

V. Dilution of color

VI. Comparison of procedures.... 368

VII. Appendix. Table 2.-Densities of pure sucrose solutions._... 370

\section{INTRODUCTION}

Peters and $\mathrm{Phelps}^{2}$ in an earlier publication, hereinafter referred to as T338, have described methods used in the preparation of sugar solutions for colorimetric analysis and have discussed the optical properties of sugar solutions, particularly with reference to the effect of turbidity upon color measurement. They found that uncontrollable errors of varying magnitude result from turbidity and that Beer's law does not hold for turbid solutions. In order to secure the necessary degree and stability of transparency, advantage was taken of the protective action of sucrose in concentrated solution toward the turbidity-producing colloids. The solutions were so prepared as to have as high a concentration of total solids or dry substance (d. s.) as was compatible with a reasonably rapid filtration rate with the filtering apparatus then available and at the same time avoid crystalization. The dry substance (d. s.) concentration was placed at about $0.70 \mathrm{~g}$ per $\mathrm{ml}$, corresponding to approximately 55 Brix. The solutions were then filtered through pads of purified asbestos.

More recent experience, especially with hard white sugars, has shown that a more satisfactory and more stable degree of transparency is obtainable for this class of sugars if the dry substance concentration be made much higher. In the present paper it is therefore recommended that this never be lower than 60 Brix (about $0.77 \mathrm{~g}$ per $\mathrm{ml}$ ) and without disadvantage may be higher. The samples of sugar product are to be dissolved and diluted with the aid of heat and 1 Presented in part before the sugar division of the American Chemical Society at Swampscott, Mass.,
September, 1928, under the title "The Spectrophotometry of White Sugars."

2 H. H. Peters and F. P. Phelps, B. S. Tech. Paper No. 338, Pt. II, March, 1927. 
filtered warm. Filtering apparatus with greater filter area than that formerly used for retaining the asbestos is also described so that the process of clarification is hastened. A method for the dilution of color with dry colorless sugar is also described.

\section{FILTERS}

Two filters with pads of purified asbestos are to be prepared before the sugar solution is made up. Various forms of filters are suitable. Among these the $25 \mathrm{ml}$ Gooch crucibles fitted with a disk of 200-mesh bolting silk, as recommended in T338, page 211, is convenient and low priced. Greater filter area may be similarly obtained by fitting a $10 \mathrm{~cm}$ glass funnel with a $4 \mathrm{~cm}$ perforated porcelain plate which is then covered with 200-mesh bolting silk as above. Because of convenience and cleanliness Jena filters in the form of cylindrical funnels, 60 or $120 \mathrm{ml}$ capacity with $4 \mathrm{~cm}$ fritted glass filter disks, have been found most satisfactory. The largest pore size, No. 1, is used for preliminary, and No. 3 which is much finer, for the final filtration. The pores of the latter frequently become clogged with fine asbestos which retards filtration. The intermediate pore size, No. 2, allows the passage of fine asbestos which, however, usually ceases after several washings with water and the filtration of 100 $\mathrm{ml}$ or less of high density sirup. This size as well as the No. 1, after careful preparation of the pads, has been successfully used for the final filtration.

When forming the pad, regardless of what form of filter is employed, the asbestos is sucked down by means of the aspirator and packed tightly by tamping with a blunt stirring rod to produce a layer about $0.5 \mathrm{~cm}$ thick. The pad is then washed several times with water (See T338, p. 271.) Equally good clarification has been obtained with $\mathrm{XXX}, \mathrm{XX}$, or A grade asbestos. The finer-fibered grades B and $\mathrm{C}$ give satisfactory filtration, but there is greater difficulty in washing out the acid used in purification. A rapid method for purifying asbestos has been described elsewhere. ${ }^{3}$

Diatomaceous earth has been used, especially in Europe, as an aid in clarifying sugar solutions for colorimetric analysis. Mastalir ${ }^{4}$ has recently shown that this material has a decided decolorizing effect upon molasses solutions.

Working with a 60 Brix solution of washed raw sugar rendered transparent by two filtrations with asbestos, the present writers twice repeated the filtration with purified standard filter cel (1 per cent by weight of dry substance) and observed the following decrease in $-\log t$ at wave length 560: Asbestos filtrate, $-\log t=0.236$; first filter cel filtrate, $-\log t=0.216$; second filter cel filtrate, $-\log t=0.166$. A corresponding decrease was observed at four other wave lengths. Because of this decolorizing action, diatomaceous earth, even in the improved forms now available, is not regarded as being suitable for the clarification of sugar solutions that are to be subjected to colorimetric analysis. Peters and Phelps (T338, p. 269), after trying the best grades of Kieselguhr then available, rejected this material because it not only decolorized but also acted selectively, causing a change in the slope of the absorption curve.

s J. F. Brewster and F. P. Phelps, J. Ind. F.ng. Chem. (Analyt. ed.), vol. 2, p. 373, 1930.

- V. Mastalir, Zcit. Zuckiud. Cechosl. liej., vol. 36, 1). 337, 1931-32. 


\section{CALCULATION OF WEIGHTS}

A generous supply of turbid solution should be available, the amount to be prepared depending upon the capacity of the optical cells employed. Time is saved by preparing a definite weight of solution having a definite, predetermined Brix which may be satisfactorily approximated by using a rough balance. No fractional gram weights are used except in the case of very dark products that require dilution of color. These will be considered in a later section. The required weights of sample and water may be calculated as follows:

$\frac{\text { Weight of solution required } \times \text { Brix of solution }}{\text { Brix of sample }}=$ weight of sample to be taken.

Weight of water to be added = weight of solution - weight of sample. The Brix of dry sugars may be assumed to be 100 . In the case of nearly dry products of relatively high purity such as 96 test raw sugars, a sufficiently close approximation results if polarization be substituted for Brix of sample in the denominator of the above fraction.

The transparency of thin liquors may be improved by the addition of an amount of sucrose, previously tested and found to be colorless, sufficient to increase the Brix to 60 or higher. The weight of water in this case remains constant and the formula for calculation may be written:

$\frac{\text { Weight of solution required } \times(100-\text { Brix of solution })}{100-\text { Brix sample }}=$ weight oî sample.

Weight of solution - weight of sample $=$ weight of sucrose to be added.

\section{PREPARATION AND FILTRATION OF THE SAMPLE}

The calculated amount of sample to provide a solution of 60 to 65 Brix is weighed in a tared flask and weights equivalent to the required amount of water are added to the pan. The flask with contents is placed in a water bath heated to $80^{\circ}$ to $90^{\circ} \mathrm{C}$. and hot distilled water is added in small quantities at a time, the flask being shaken to promote quick solution and to insure a high concentration. To guard against over dilution the flask dried on the outside is occasionally placed on the balance pan.

When solution is complete and the required amount of water has been added, purified asbestos (grade $A$ ) in amount equal to about 0.5 per cent of the solution is added. The flask is then closed with a clean rubber stopper, and vigorously shaken. The flask is returned to the warm bath and the preliminary filter is heated by rinsing with hot distilled water which is drawn off as thoroughly as possible by suction. A few milliters of the warm solution are poured on the pad and drawn through to displace water. The main portion of the solution, or as much as the filter will hold, is added and after a few drops have filtered the suction is stopped and a clean receiver is substituted. The pads are to be kept covered with solution during filtration, at the end of which the suction is stopped before the pad becomes uncovered. The receiver is detached and returned to the bath while the second filter is being rinsed with hot water and drained. 
The second filtration is performed exactly as the first, no asbestos being added to the first filtrate. The main portion of the filtrate is collected in a clean receiver. This filtrate is cooled and adhering water wiped from the neck of the bottle which is then closed with a clean, dry stopper and shaken to mix the contents thoroughly. The refractometric Brix of the optically clear filtrate is determined and $c$ ( $=\mathrm{g}$ dry substance per $\mathrm{ml}$ ) obtained by reference to Table 2 (Appendix). The solution is now ready for colorimetric examination. With properly prepared solutions and filters further filtration is of doubtful value unless some scattered particles of asbestos have passed through. If the second filtrate appears turbid the best practice is to prepare a new solution with increased dry substance concentration. Jena filters are easily cleansed by removing the pads and drawing water and air through the pores by means of the aspirator connected alternately to the mouth and the stem of the funnel. This dislodges asbestos fibres, which may be poured out. Treatment of the glass filters with chromic-sulphuric acid mixture removes waxy or greasy film deposited by sugar solutions.

\section{DILUTION OF COLOR}

Directions are given in T338, pp. 277 and 285-286, for the dilution of very dark sugar solutions with colorless sirup in order that good photometric readings may be made and to insure a high degree of transparency. Instead of employing for this purpose a stock sirup, which may be subject to decomposition and change in storage, a high-grade, nearly colorless solid sugar may be used in the following manner: One hundred grams of the solid sugar are weighed in a tared flask. A small amount of the colored sample whose dry substance content has been determined is introduced and the whole reweighed. All these weighings should be accurate to the nearest milligram in order that the proportion of colored dry substance may be calculated. The mixture is dissolved and diluted to 60 to 65 Brix exactly as described in the foregoing section. Sugar of the quality of the highest grade refinery tablets may be used as diluent and for accurate results its absorbancy may be deducted. The specific absorptive index of good tablet sugars is less than 0.005 at wave length 560 and in many cases this may be ignored as not affecting the final results. The reference at the head of this section is to be consulted for methods of calculating colored dry substance in the mixture.

\section{COMPARISON OF PROCEDURES}

In Table 1 are given for comparison results obtained by varying the dry substance concentration and the temperature at which the samples were dissolved and filtered. Four types of sugar products were used, each diluted to three different d. s. concentrations designated in column 2 as $\mathrm{A}, \mathrm{B}$, and $\mathrm{C}$, corresponding, respectively, to 55,60 , and 65 Brix, the approximate concentration before filtration. The final concentration after filtration and cooling is given as refractometric Brix in column 3 , and as the corresponding $c$ in $g$ per $\mathrm{ml}$ in column 4 . In column 5 is given the specific absorptive index, $-\log t$, at $\lambda 560 \mathrm{~m} \mu$ of each solution. In column 6 are shown the differences $(-\log t \mathrm{~A})-(-\log t \mathrm{~B})$ and $(-\log t \mathrm{C})-(-\log t \mathrm{~B})$ expressed as per cent-log $t$ B. In column 7 the approximate 
temperatures at which the solutions were prepared are given. The solutions prepared at $40^{\circ}$ were filtered at room temperature, while those prepared at $70^{\circ}$ were filtered at $70^{\circ}$.

The influence of dry substance concentration is to be seen by reference to columns 5 and 6 . Regardless of the temperature at which the solutions were prepared, the highest absorption value for any given product occurs in the solution having the lowest density and is attributed to the presence of turbidity in these solutions that is not removable by the filtration method used. (Compare T388, p. 280.)

Samples 8 and 9 were prepared by dispersing the molasses in very pure sucrose solution as described in Section V. The proportion of molasses dry substance in total dry substance varied from 0.88 to 1.13 per cent and the general agreement among the results demonstrates the effectiveness of sucrose in preventing turbidity. Optical disturbance due to crystallization in 70 Brix sugar solutions has never been encountered and it is therefore inferred that many high density refinery or factory liquors may be prepared for colorimetric analysis without further dilution.

TABLE 1.-Comparison of procedures

I. PLANTATION GRANULATED SUGARS

\begin{tabular}{|c|c|c|c|c|c|c|}
\hline Sample No. & Designation of solution & $\begin{array}{l}\text { Brix by } \\
\text { refrac- } \\
\text { tometer }\end{array}$ & $c$ in $\mathrm{g} / \mathrm{ml}$ & $\begin{array}{c}-\log t \\
\lambda 560\end{array}$ & $\begin{array}{l}\text { Differ- } \\
\text { ence per } \\
\text { cent on } \\
-\log t \mathrm{~B}\end{array}$ & $\begin{array}{l}\text { Approxi- } \\
\text { mate tem- } \\
\text { perature } \\
\text { of solu- } \\
\text { tion }\end{array}$ \\
\hline 1 & 2 & 3 & 4 & 5 & 6 & 7 \\
\hline & - n & $\begin{array}{l}55.2 \\
61.3 \\
66.4\end{array}$ & $\begin{array}{r}0.6948 \\
.7933 \\
.8800\end{array}$ & $\begin{array}{r}0.0213 \\
0.0193 \\
.0199\end{array}$ & $\begin{array}{r}+10.40 \\
+3.11\end{array}$ & $\begin{array}{r}\circ C . \\
40 \\
70 \\
70\end{array}$ \\
\hline & $\left\{\begin{array}{l}\mathrm{A}-\mathrm{W} \\
\mathrm{B} \\
\mathrm{C}\end{array}\right.$ & $\begin{array}{l}56.0 \\
61.4 \\
66.3\end{array}$ & $\begin{array}{l}.7074 \\
.7940 \\
.8780\end{array}$ & $\begin{array}{l}.0458 \\
.0442 \\
.0440\end{array}$ & $\begin{array}{r}+3.62 \\
-.45\end{array}$ & $\begin{array}{l}40 \\
40 \\
40\end{array}$ \\
\hline
\end{tabular}

II. WASHED RATV SUGARS

\begin{tabular}{|c|c|c|c|c|c|c|}
\hline 3. & 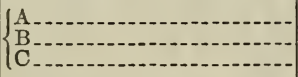 & $\begin{array}{l}55.1 \\
60.7 \\
67.0\end{array}$ & $\begin{array}{r}0.6533 \\
.7834 \\
.8064\end{array}$ & $\begin{array}{l}0.349 \\
.275 \\
.276\end{array}$ & $\begin{array}{r}+26.29 \\
+.36\end{array}$ & $\begin{array}{l}70 \\
70 \\
70\end{array}$ \\
\hline 4. & $\left\{\begin{array}{l}\mathrm{A} \\
\mathrm{B} \\
\mathrm{C}\end{array}\right.$ & $\begin{array}{l}55.4 \\
61.0 \\
68.8\end{array}$ & $\begin{array}{l}.6979 \\
.7883 \\
.9217\end{array}$ & $\begin{array}{l}.300 \\
.212 \\
.208\end{array}$ & $\begin{array}{r}+41.51 \\
-1.89\end{array}$ & $\begin{array}{l}40 \\
40 \\
70\end{array}$ \\
\hline 5. & $\left\{\begin{array}{l}\mathrm{A} \\
\mathrm{B} \\
\mathrm{B} \\
\mathrm{C}\end{array}\right.$ & $\begin{array}{l}57.5 \\
60.1 \\
69.6\end{array}$ & $\begin{array}{l}.7313 \\
.7735 \\
.9359\end{array}$ & $\begin{array}{l}.244 \\
.232 \\
.234\end{array}$ & $\begin{array}{r}+5.18 \\
+.85\end{array}$ & $\begin{array}{l}70 \\
70 \\
70\end{array}$ \\
\hline
\end{tabular}

III. RAW SUGARS (UNWASHED)

\begin{tabular}{|c|c|c|c|c|c|c|}
\hline 6 & $\left\{\begin{array}{l}\mathrm{A}_{\mathrm{N}} \\
\mathrm{B} \\
\mathrm{C}\end{array}\right.$ & $\begin{array}{l}55.2 \\
61.4 \\
71.8\end{array}$ & $\begin{array}{r}0.6948 \\
.7949 \\
.9754\end{array}$ & $\begin{array}{l}1.224 \\
1.120 \\
1.105\end{array}$ & $\begin{array}{r}+9.29 \\
-1.34\end{array}$ & $\begin{array}{l}40 \\
70 \\
70\end{array}$ \\
\hline & 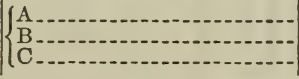 & $\begin{array}{l}55.1 \\
61.4 \\
70.3\end{array}$ & $\begin{array}{r}.6932 \\
.7949 \\
.9483\end{array}$ & $\begin{array}{l}1.201 \\
1.001 \\
1.000\end{array}$ & $\begin{array}{r}+19.99 \\
-.10\end{array}$ & $\begin{array}{l}70 \\
70 \\
70\end{array}$ \\
\hline
\end{tabular}

IV. MOLASSES

\begin{tabular}{|c|c|c|c|c|c|c|}
\hline 8. & 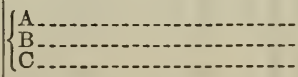 & $\begin{array}{l}55.7 \\
60.5 \\
66.1\end{array}$ & $\begin{array}{r}0.7027 \\
.7801 \\
.8750\end{array}$ & $\begin{array}{l}33.10 \\
32.30 \\
32.82\end{array}$ & $\begin{array}{r}+2.48 \\
+1.55\end{array}$ & $\begin{array}{l}70 \\
70 \\
70\end{array}$ \\
\hline 9 & $\left\{\begin{array}{l}\mathrm{A} \\
\mathrm{B} \\
\mathrm{C}-\mathrm{n}\end{array}\right.$ & $\begin{array}{l}56.2 \\
61.8 \\
66.2\end{array}$ & $\begin{array}{l}.7106 \\
.8016 \\
.8763\end{array}$ & $\begin{array}{l}53.00 \\
52.70 \\
53.10\end{array}$ & $\begin{array}{l}+.57 \\
+.76\end{array}$ & $\begin{array}{l}70 \\
70 \\
70\end{array}$ \\
\hline
\end{tabular}




\section{APPENDIX. TABLE 2.-DENSITIES OF PURE SUCROSE SOLUTIONS}

This table is an expansion of Table 2, B. S. Tech. Paper No. 338, page 304, and gives values corresponding to degrees Brix to cover the higher densities recommended. It is to be used for finding values of $c$ (g dry substance per $\mathrm{ml}$ ) corresponding to degrees Brix, density, or apparent specific gravity of sugar solutions from analy tical data. The tabulated values were derived in the manner already fully described in the publication referred to above.

TABLE 2.-Densities of pure sucrose solutions

\begin{tabular}{|c|c|c|c|c|c|c|c|c|c|}
\hline $\begin{array}{l}\text { Brix } \\
\text { or true } \\
\text { per cent } \\
\text { d. s. by } \\
\text { weight }\end{array}$ & $\begin{array}{c}\text { True } \\
\text { density } \\
\text { (Plato) } \\
20^{\circ}\end{array}$ & $\begin{array}{l}\text { Appar- } \\
\text { ont den- } \\
\text { sity } 20^{\circ}\end{array}$ & $\begin{array}{l}\text { Appar- } \\
\text { ent spe- } \\
\text { cific } \\
\text { gravity } \\
20^{\circ} / 20^{\circ}\end{array}$ & $\begin{array}{l}\text { g sucrose } \\
\text { per } 100 \mathrm{ml} \\
\text { weight } \\
\text { in vacuo }\end{array}$ & $\begin{array}{c}\text { Brix } \\
\text { or true } \\
\text { per cent } \\
\text { d. s. by } \\
\text { weight }\end{array}$ & $\begin{array}{c}\text { True } \\
\text { density } \\
\text { (Plato) } \\
20^{\circ}\end{array}$ & $\begin{array}{l}\text { Appar- } \\
\text { ent den- } \\
\text { sity } 20^{\circ}\end{array}$ & $\begin{array}{l}\text { A ppar- } \\
\text { ent spe- } \\
\text { cific } \\
\text { gravity } \\
20^{\circ} / 20^{\circ}\end{array}$ & $\begin{array}{l}\text { g sucrose } \\
\text { per } 100 \mathrm{ml} \\
\text { weight } \\
\text { in vacuo }\end{array}$ \\
\hline 1 & 2 & 3 & 4 & 5 & 1 & 2 & 3 & 4 & 5 \\
\hline $\begin{array}{l}59.0 \\
59.1 \\
59.2 \\
59.3 \\
59.4\end{array}$ & $\begin{array}{r}1.28059 \\
118 \\
176 \\
235 \\
294\end{array}$ & $\begin{array}{r}\text { 1. } 27958 \\
1.28017 \\
075 \\
134 \\
193\end{array}$ & $\begin{array}{r}1.28320 \\
379 \\
437 \\
497 \\
556\end{array}$ & $\begin{array}{r}75.555 \\
.718 \\
.880 \\
76.043 \\
.207\end{array}$ & $\begin{array}{l}64.0 \\
64.1 \\
64.2 \\
64.3 \\
64.4\end{array}$ & $\begin{array}{r}1.31028 \\
089 \\
149 \\
209 \\
270\end{array}$ & $\begin{array}{r}1.30927 \\
988 \\
1.31048 \\
108 \\
169\end{array}$ & $\begin{array}{r}1.31297 \\
359 \\
418 \\
479 \\
540\end{array}$ & $\begin{array}{r}83.858 \\
84.028 \\
.198 \\
.367 \\
.538\end{array}$ \\
\hline $\begin{array}{l}59.5 \\
59.6 \\
59.7 \\
59.8 \\
59.9\end{array}$ & $\begin{array}{l}352 \\
411 \\
469 \\
528 \\
587\end{array}$ & $\begin{array}{l}251 \\
309 \\
367 \\
426 \\
485\end{array}$ & $\begin{array}{l}614 \\
672 \\
731 \\
789 \\
849\end{array}$ & $\begin{array}{r}.369 \\
.533 \\
.696 \\
.860 \\
77.024\end{array}$ & $\begin{array}{l}64.5 \\
64.6 \\
64.7 \\
64.8 \\
64.9\end{array}$ & $\begin{array}{l}330 \\
391 \\
452 \\
512 \\
573\end{array}$ & $\begin{array}{l}229 \\
290 \\
350 \\
412 \\
473\end{array}$ & $\begin{array}{l}600 \\
661 \\
723 \\
784 \\
845\end{array}$ & $\begin{array}{r}.708 \\
.879 \\
85.049 \\
.220 \\
.391\end{array}$ \\
\hline $\begin{array}{l}60.0 \\
60.1 \\
60.2 \\
60.3 \\
60.4\end{array}$ & $\begin{array}{r}1.28646 \\
704 \\
763 \\
822 \\
881\end{array}$ & $\begin{array}{r}1.28544 \\
602 \\
661 \\
720 \\
779\end{array}$ & $\begin{array}{r}1.28908 \\
966 \\
1.29025 \\
084 \\
143\end{array}$ & $\begin{array}{r}77.188 \\
.351 \\
.515 \\
.680 \\
.844\end{array}$ & $\begin{array}{l}65.0 \\
65.1 \\
65.2 \\
65.3 \\
65.4\end{array}$ & $\begin{array}{r}1.31633 \\
694 \\
755 \\
816 \\
877\end{array}$ & $\begin{array}{r}1.31533 \\
594 \\
655 \\
716 \\
777\end{array}$ & $\begin{array}{r}1.31905 \\
966 \\
1.32028 \\
089 \\
150\end{array}$ & $\begin{array}{r}85.561 \\
.733 \\
.904 \\
86.076 \\
.248\end{array}$ \\
\hline $\begin{array}{l}60.5 \\
60.6 \\
60.7 \\
60.8 \\
60.9\end{array}$ & $\begin{array}{r}940 \\
999 \\
1.29058 \\
117 \\
176\end{array}$ & $\begin{array}{r}838 \\
897 \\
956 \\
1.29015 \\
074\end{array}$ & $\begin{array}{l}203 \\
262 \\
321 \\
380 \\
439\end{array}$ & $\begin{array}{r}78.009 \\
.173 \\
.339 \\
.503 \\
.668\end{array}$ & $\begin{array}{l}65.5 \\
65.6 \\
65.7 \\
65.8 \\
65.9\end{array}$ & $\begin{array}{r}937 \\
998 \\
1.32059 \\
120 \\
181\end{array}$ & $\begin{array}{r}837 \\
898 \\
959 \\
1.32019 \\
081\end{array}$ & $\begin{array}{l}210 \\
271 \\
332 \\
393 \\
455\end{array}$ & $\begin{array}{r}.419 \\
.591 \\
.763 \\
.935 \\
87.107\end{array}$ \\
\hline $\begin{array}{l}61.0 \\
61.1 \\
61.2 \\
61.3 \\
61.4\end{array}$ & $\begin{array}{r}1.29235 \\
295 \\
354 \\
413 \\
472\end{array}$ & $\begin{array}{r}29133 \\
193 \\
252 \\
311 \\
370\end{array}$ & $\begin{array}{r}1.29498 \\
559 \\
618 \\
677 \\
736\end{array}$ & $\begin{array}{r}78.833 \\
.999 \\
79.165 \\
.330 \\
.496\end{array}$ & $\begin{array}{l}66.0 \\
66.1 \\
66.2 \\
66.3 \\
66.4\end{array}$ & $\begin{array}{r}1.32243 \\
304 \\
365 \\
426 \\
487\end{array}$ & $\begin{array}{r}1.32142 \\
203 \\
264 \\
325 \\
385\end{array}$ & $\begin{array}{r}1.32516 \\
577 \\
638 \\
699 \\
759\end{array}$ & $\begin{array}{r}87.280 \\
.453 \\
.626 \\
.798 \\
.971\end{array}$ \\
\hline $\begin{array}{l}61.5 \\
61.6 \\
61.7 \\
61.8 \\
61.9\end{array}$ & $\begin{array}{l}532 \\
591 \\
651 \\
710 \\
770\end{array}$ & $\begin{array}{l}430 \\
489 \\
548 \\
608 \\
667\end{array}$ & $\begin{array}{r}796 \\
855 \\
915 \\
975 \\
1.30034\end{array}$ & $\begin{array}{r}.662 \\
.828 \\
.995 \\
80.161 \\
.328\end{array}$ & $\begin{array}{l}66.5 \\
66.6 \\
66.7 \\
66.8 \\
66.9\end{array}$ & $\begin{array}{l}548 \\
610 \\
671 \\
733 \\
794\end{array}$ & $\begin{array}{l}446 \\
509 \\
570 \\
632 \\
693\end{array}$ & $\begin{array}{r}820 \\
884 \\
945 \\
1.33007 \\
068\end{array}$ & $\begin{array}{r}88.142 \\
.318 \\
.492 \\
.666 \\
.839\end{array}$ \\
\hline $\begin{array}{l}62.0 \\
62.1 \\
62.2 \\
62.3 \\
62.4\end{array}$ & $\begin{array}{r}1.29829 \\
889 \\
948 \\
1.30008 \\
068\end{array}$ & $\begin{array}{r}1.29726 \\
786 \\
845 \\
905 \\
966\end{array}$ & $\begin{array}{r}1.30093 \\
153 \\
212 \\
273 \\
234\end{array}$ & $\begin{array}{r}80.494 \\
.661 \\
.828 \\
.995 \\
81.162\end{array}$ & $\begin{array}{l}67.0 \\
67.1 \\
67.2 \\
67.3 \\
67.4\end{array}$ & $\begin{array}{r}1.32855 \\
917 \\
979 \\
1.33040 \\
102\end{array}$ & $\begin{array}{r}1.32754 \\
816 \\
878 \\
939 \\
1.33001\end{array}$ & $\begin{array}{r}1.33129 \\
192 \\
254 \\
315 \\
377\end{array}$ & $\begin{array}{r}89.012 \\
.187 \\
.361 \\
.536 \\
.711\end{array}$ \\
\hline $\begin{array}{l}62.5 \\
62.6 \\
62.7 \\
62.8 \\
62.9\end{array}$ & $\begin{array}{l}127 \\
187 \\
247 \\
307 \\
367\end{array}$ & $\begin{array}{r}1.30025 \\
085 \\
145 \\
205 \\
265\end{array}$ & $\begin{array}{l}393 \\
453 \\
513 \\
573 \\
633\end{array}$ & $\begin{array}{r}.329 \\
.497 \\
.665 \\
.833 \\
82.001\end{array}$ & $\begin{array}{l}67.5 \\
67.6 \\
67.7 \\
67.8 \\
67.9\end{array}$ & $\begin{array}{l}163 \\
225 \\
287 \\
349 \\
410\end{array}$ & $\begin{array}{l}062 \\
124 \\
186 \\
248 \\
309\end{array}$ & $\begin{array}{l}438 \\
500 \\
562 \\
625 \\
656\end{array}$ & $\begin{array}{r}90.885 \\
.060 \\
.235 \\
.411 \\
.585\end{array}$ \\
\hline \begin{tabular}{l|}
63.0 \\
63.1 \\
63.2 \\
63.3 \\
63.4
\end{tabular} & $\begin{array}{r}1.30427 \\
487 \\
547 \\
607 \\
667\end{array}$ & $\begin{array}{r}30325 \\
385 \\
446 \\
506 \\
566\end{array}$ & $\begin{array}{r}1.30694 \\
754 \\
815 \\
875 \\
936\end{array}$ & $\begin{array}{r}82.169 \\
.337 \\
.506 \\
.674 \\
.843\end{array}$ & $\begin{array}{l}68.0 \\
68.1 \\
68.2 \\
68.3 \\
68.4\end{array}$ & $\begin{array}{r}1.33472 \\
534 \\
596 \\
658 \\
720\end{array}$ & $\begin{array}{r}1.33371 \\
433 \\
495 \\
557 \\
619\end{array}$ & $\begin{array}{r}\text { 1. } 33748 \\
810 \\
872 \\
935 \\
997\end{array}$ & $\begin{array}{r}90.761 \\
.937 \\
91.112 \\
.288 \\
.464\end{array}$ \\
\hline $\begin{array}{l}63.5 \\
63.6 \\
63.7 \\
63.8 \\
63.9\end{array}$ & $\begin{array}{l}727 \\
787 \\
848 \\
908 \\
968\end{array}$ & $\begin{array}{l}626 \\
686 \\
747 \\
807 \\
867\end{array}$ & $\begin{array}{r}994 \\
1.31055 \\
117 \\
177 \\
237\end{array}$ & $\begin{array}{r}83.012 \\
.180 \\
.350 \\
.519 \\
.688\end{array}$ & $\begin{array}{l}68.5 \\
68.6 \\
68.7 \\
68.8 \\
68.9\end{array}$ & $\begin{array}{r}782 \\
844 \\
906 \\
968 \\
\text { 1. } 34031\end{array}$ & $\begin{array}{l}681 \\
743 \\
805 \\
867 \\
930\end{array}$ & $\begin{array}{r}1.34059 \\
121 \\
183 \\
245 \\
309\end{array}$ & $\begin{array}{r}.641 \\
.817 \\
.993 \\
92.169 \\
.347\end{array}$ \\
\hline
\end{tabular}


TABLE 2.-Densities of pure sucrose solutions-Continued

\begin{tabular}{|c|c|c|c|c|c|c|c|c|c|}
\hline $\begin{array}{l}\text { Brix } \\
\text { or true } \\
\text { per cent } \\
\text { d. s. by } \\
\text { weight }\end{array}$ & $\begin{array}{c}\text { True } \\
\text { density } \\
\text { (Plato) } \\
20^{\circ}\end{array}$ & $\begin{array}{l}\text { Appar- } \\
\text { ent den- } \\
\text { sity } 20^{\circ}\end{array}$ & $\begin{array}{l}\text { Appar- } \\
\text { ent spe- } \\
\text { cific } \\
\text { gravity } \\
20^{\circ} / 20^{\circ}\end{array}$ & $\begin{array}{l}\text { g sucrose } \\
\text { per } 100 \mathrm{ml} \\
\text { weight } \\
\text { in vacuo }\end{array}$ & $\begin{array}{l}\text { Brix } \\
\text { or true } \\
\text { per cent } \\
\text { d.s. by } \\
\text { weight }\end{array}$ & $\begin{array}{c}\text { True } \\
\text { density } \\
\text { (Plato) } \\
20^{\circ}\end{array}$ & $\begin{array}{l}\text { A ppar- } \\
\text { ent den- } \\
\text { sity } 20^{\circ}\end{array}$ & $\begin{array}{c}\text { Appar- } \\
\text { ent spe- } \\
\text { cific } \\
\text { gravity } \\
20^{\circ} / 20^{\circ}\end{array}$ & $\begin{array}{l}\text { g sucrose } \\
\text { per } 100 \mathrm{ml} \\
\text { weight } \\
\text { in vacuo }\end{array}$ \\
\hline 1 & 2 & 3 & 4 & 5 & 1 & 2 & 3 & 4 & 5 \\
\hline 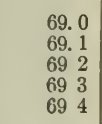 & $\begin{array}{r}1.34093 \\
155 \\
217 \\
280 \\
342\end{array}$ & $\begin{array}{r}\text { 1. } 33992 \\
1.34054 \\
116 \\
179 \\
241\end{array}$ & $\begin{array}{r}1.34371 \\
433 \\
495 \\
558 \\
621\end{array}$ & $\begin{array}{r}92.524 \\
.701 \\
.878 \\
93.056 \\
.233\end{array}$ & $\begin{array}{l}75.5 \\
75.6 \\
75.7 \\
75.8 \\
75.9\end{array}$ & $\begin{array}{r}1.38220 \\
285 \\
350 \\
415 \\
480\end{array}$ & $\begin{array}{r}1.38119 \\
184 \\
249 \\
314 \\
379\end{array}$ & $\begin{array}{r}1.38510 \\
-575 \\
640 \\
705 \\
770\end{array}$ & $\begin{array}{r}104.356 \\
.543 \\
.731 \\
.919 \\
105.106\end{array}$ \\
\hline $\begin{array}{ll}69 & 5 \\
69 & 6 \\
69.7 \\
69.8 \\
69.9\end{array}$ & $\begin{array}{l}405 \\
467 \\
530 \\
592 \\
655\end{array}$ & $\begin{array}{l}304 \\
366 \\
429 \\
491 \\
554\end{array}$ & $\begin{array}{l}684 \\
746 \\
809 \\
871 \\
934\end{array}$ & $\begin{array}{r}.411 \\
.589 \\
.767 \\
.945 \\
94.123\end{array}$ & $\begin{array}{l}76.0 \\
76.1 \\
76.2 \\
76.3 \\
76.4\end{array}$ & $\begin{array}{r}1.38545 \\
610 \\
675 \\
740 \\
805\end{array}$ & $\begin{array}{r}1.38444 \\
510 \\
575 \\
640 \\
705\end{array}$ & $\begin{array}{r}1.38835 \\
902 \\
967 \\
1.39032 \\
097\end{array}$ & $\begin{array}{r}105.294 \\
.482 \\
.670 \\
.859 \\
106.047\end{array}$ \\
\hline $\begin{array}{l}70.0 \\
70.1 \\
70.2 \\
70.3 \\
70.4\end{array}$ & $\begin{array}{r}1.34717 \\
780 \\
843 \\
906 \\
968\end{array}$ & $\begin{array}{r}1.34616 \\
679 \\
742 \\
805 \\
867\end{array}$ & $\begin{array}{r}1.34997 \\
1.35050 \\
123 \\
186 \\
248\end{array}$ & $\begin{array}{r}94.302 \\
.481 \\
.660 \\
.839 \\
95.017\end{array}$ & $\begin{array}{l}76.5 \\
76.6 \\
76.7 \\
76.8 \\
76.9\end{array}$ & $\begin{array}{r}870 \\
935 \\
1.39000 \\
065 \\
130\end{array}$ & $\begin{array}{r}770 \\
835 \\
900 \\
965 \\
1.39030\end{array}$ & $\begin{array}{l}162 \\
228 \\
293 \\
358 \\
423\end{array}$ & $\begin{array}{l}.236 \\
.424 \\
.613 \\
.802 \\
.991\end{array}$ \\
\hline $\begin{array}{l}70.5 \\
70.6 \\
70.7 \\
70.8 \\
70.9\end{array}$ & $\begin{array}{r}1.35031 \\
094 \\
157 \\
220 \\
233\end{array}$ & $\begin{array}{r}930 \\
993 \\
1.35056 \\
119 \\
182\end{array}$ & $\begin{array}{l}311 \\
375 \\
438 \\
501 \\
564\end{array}$ & $\begin{array}{l}.197 \\
.376 \\
.556 \\
.736 \\
.916\end{array}$ & $\begin{array}{l}77.0 \\
77.1 \\
77.2 \\
77.3 \\
77.4\end{array}$ & $\begin{array}{r}1.39196 \\
261 \\
326 \\
392 \\
457\end{array}$ & $\begin{array}{r}1.39096 \\
161 \\
225 \\
291 \\
356\end{array}$ & $\begin{array}{r}1.39489 \\
554 \\
619 \\
685 \\
750\end{array}$ & $\begin{array}{r}107.181 \\
.370 \\
.560 \\
.750 \\
.940\end{array}$ \\
\hline $\begin{array}{l}71.0 \\
71.1 \\
71.2 \\
71.3 \\
71.4\end{array}$ & $\begin{array}{r}1.35346 \\
409 \\
472 \\
535 \\
598\end{array}$ & $\begin{array}{r}1.35245 \\
308 \\
371 \\
434 \\
498\end{array}$ & $\begin{array}{r}1.35627 \\
691 \\
754 \\
817 \\
881\end{array}$ & $\begin{array}{r}96.096 \\
.276 \\
.456 \\
.636 \\
.817\end{array}$ & & $\begin{array}{l}523 \\
588 \\
654 \\
719 \\
785\end{array}$ & $\begin{array}{l}422 \\
488 \\
554 \\
619 \\
685\end{array}$ & $\begin{array}{r}816 \\
882 \\
949 \\
1.40014 \\
080\end{array}$ & $\begin{array}{r}108.130 \\
.320 \\
.511 \\
.701 \\
.892\end{array}$ \\
\hline $\begin{array}{l}71.5 \\
71.6 \\
71.7 \\
71.8 \\
71.9\end{array}$ & $\begin{array}{l}661 \\
725 \\
788 \\
851 \\
914\end{array}$ & $\begin{array}{l}561 \\
625 \\
688 \\
751 \\
814\end{array}$ & $\begin{array}{r}944 \\
1.36008 \\
072 \\
135 \\
198\end{array}$ & $\begin{array}{r}.998 \\
97.179 \\
.360 \\
.541 \\
.722\end{array}$ & $\begin{array}{l}78.0 \\
78.1 \\
78.2 \\
78.3 \\
78.4\end{array}$ & $\begin{array}{r}1.39851 \\
916 \\
982 \\
1.40048 \\
113\end{array}$ & $\begin{array}{r}1.39751 \\
816 \\
882 \\
548 \\
1.40013\end{array}$ & $\begin{array}{r}1.40146 \\
211 \\
277 \\
344 \\
409\end{array}$ & $\begin{array}{r}109.084 \\
.274 \\
.466 \\
.657 \\
.848\end{array}$ \\
\hline $\begin{array}{l}72.0 \\
72.1 \\
72.2 \\
72.3 \\
72.4\end{array}$ & $\begin{array}{r}1.35978 \\
1.36041 \\
105 \\
168 \\
232\end{array}$ & $\begin{array}{r}1.35877 \\
940 \\
1.36004 \\
067 \\
131\end{array}$ & $\begin{array}{r}1.36261 \\
324 \\
389 \\
452 \\
516\end{array}$ & $\begin{array}{r}97.904 \\
98.085 \\
.268 \\
.449 \\
.632\end{array}$ & & $\begin{array}{l}179 \\
245 \\
311 \\
377 \\
443\end{array}$ & 079 & $\begin{array}{l}475 \\
541 \\
607 \\
674 \\
740\end{array}$ & $\begin{array}{r}110.041 \\
.232 \\
.425 \\
.617 \\
.809\end{array}$ \\
\hline $\begin{array}{l}72.5 \\
72.6 \\
72.7 \\
72.8 \\
72.9\end{array}$ & $\begin{array}{l}295 \\
359 \\
423 \\
486 \\
550\end{array}$ & $\begin{array}{l}194 \\
258 \\
322 \\
385 \\
450\end{array}$ & $\begin{array}{l}579 \\
643 \\
707 \\
771 \\
836\end{array}$ & $\begin{array}{r}.814 \\
.997 \\
99.179 \\
.362 \\
.545\end{array}$ & $\begin{array}{l}79.0 \\
79.1 \\
79.2 \\
79.3 \\
79.4\end{array}$ & $\begin{array}{r}1.40509 \\
575 \\
641 \\
707 \\
774\end{array}$ & $\begin{array}{r}1.40409 \\
475 \\
541 \\
607 \\
674\end{array}$ & $\begin{array}{r}1.40806 \\
872 \\
938 \\
1.41005 \\
072\end{array}$ & $\begin{array}{r}111.002 \\
.195 \\
.388 \\
.581 \\
.775\end{array}$ \\
\hline $\begin{array}{l}73.0 \\
73.1 \\
73.2 \\
73.3 \\
73.4\end{array}$ & $\begin{array}{r}1.36614 \\
678 \\
742 \\
805 \\
869\end{array}$ & $\begin{array}{r}1.36514 \\
578 \\
642 \\
705 \\
769\end{array}$ & $\begin{array}{r}1.36900 \\
964 \\
1.37028 \\
092 \\
156\end{array}$ & $\begin{array}{r}99.728 \\
100.095 \\
.278 \\
.462\end{array}$ & $\begin{array}{l}79.5 \\
79.6 \\
79.7 \\
79.8 \\
79.9\end{array}$ & $\begin{array}{r}840 \\
906 \\
972 \\
1.41039 \\
105\end{array}$ & $\begin{array}{r}740 \\
806 \\
872 \\
939 \\
1.41005\end{array}$ & $\begin{array}{l}138 \\
204 \\
270 \\
337 \\
404\end{array}$ & $\begin{array}{r}.968 \\
112.161 \\
.354 \\
.549 \\
.743\end{array}$ \\
\hline $\begin{array}{l}73.5 \\
73.6 \\
73.7 \\
73.8 \\
73.9\end{array}$ & $\begin{array}{r}933 \\
997 \\
1.37061 \\
125 \\
189\end{array}$ & $\begin{array}{r}833 \\
896 \\
960 \\
1.37024 \\
088\end{array}$ & $\begin{array}{l}220 \\
283 \\
347 \\
411 \\
476\end{array}$ & $\begin{array}{r}.646 \\
.827 \\
101.014 \\
.198 \\
.383\end{array}$ & & $\begin{array}{r}1.41172 \\
238 \\
304 \\
371 \\
437\end{array}$ & $\begin{array}{r}1.41072 \\
138 \\
204 \\
271 \\
337\end{array}$ & $\begin{array}{r}1.41471 \\
537 \\
603 \\
670 \\
737\end{array}$ & $\begin{array}{r}112.938 \\
113.131 \\
.326 \\
.521 \\
.715\end{array}$ \\
\hline $\begin{array}{l}74.0 \\
74.1 \\
74.2 \\
74.3 \\
74.4\end{array}$ & $\begin{array}{r}1.37254 \\
318 \\
382 \\
446 \\
511\end{array}$ & $\begin{array}{r}1.37153 \\
217 \\
281 \\
345 \\
410\end{array}$ & $\begin{array}{r}1.37541 \\
605 \\
669 \\
733 \\
798\end{array}$ & $\begin{array}{r}101.568 \\
.753 \\
.937 \\
102.122 \\
.308\end{array}$ & $\begin{array}{l}80.5 \\
80.6 \\
80.7 \\
80.8 \\
80.9\end{array}$ & $\begin{array}{l}504 \\
571 \\
637 \\
704 \\
771\end{array}$ & $\begin{array}{l}404 \\
472 \\
537 \\
604 \\
671\end{array}$ & $\begin{array}{r}804 \\
872 \\
937 \\
1.42004 \\
072\end{array}$ & $\begin{array}{r}.911 \\
114.106 \\
.301 \\
.497 \\
.692\end{array}$ \\
\hline $\begin{array}{l}74.5 \\
74.6 \\
74.7 \\
74.8 \\
74.9\end{array}$ & $\begin{array}{l}575 \\
639 \\
704 \\
768 \\
833\end{array}$ & $\begin{array}{l}475 \\
539 \\
604 \\
668 \\
733\end{array}$ & $\begin{array}{r}864 \\
928 \\
993 \\
1.38057 \\
122\end{array}$ & $\begin{array}{r}.493 \\
.679 \\
.865 \\
103.050 \\
.237\end{array}$ & $\begin{array}{l}81.0 \\
81.1 \\
81.2 \\
81.3 \\
81.4\end{array}$ & $\begin{array}{r}1.41837 \\
904 \\
971 \\
1.42038 \\
105\end{array}$ & $\begin{array}{r}1.41737 \\
804 \\
871 \\
938 \\
1.42005\end{array}$ & $\begin{array}{r}1.42138 \\
205 \\
272 \\
339 \\
906\end{array}$ & $\begin{array}{r}114.888 \\
115.084 \\
.280 \\
.477 \\
.673\end{array}$ \\
\hline $\begin{array}{l}75.0 \\
75.1 \\
75.2 \\
75.3 \\
75.4\end{array}$ & $\begin{array}{r}1.37897 \\
962 \\
1.38026 \\
091 \\
156\end{array}$ & $\begin{array}{r}1.37797 \\
862 \\
926 \\
991 \\
1.38055\end{array}$ & $\begin{array}{r}1.38187 \\
252 \\
316 \\
381 \\
445\end{array}$ & $\begin{array}{r}103.423 \\
.609 \\
.796 \\
.983 \\
104.170\end{array}$ & $\begin{array}{l}81.5 \\
81.6 \\
81.7 \\
81.8 \\
81.9\end{array}$ & $\begin{array}{l}172 \\
239 \\
306 \\
373 \\
440\end{array}$ & $\begin{array}{l}072 \\
139 \\
206 \\
273 \\
340\end{array}$ & $\begin{array}{l}474 \\
541 \\
608 \\
675 \\
742\end{array}$ & $\begin{array}{r}.870 \\
116.067 \\
.264 \\
.461 \\
.658\end{array}$ \\
\hline
\end{tabular}


TABLE 2.-Densities of pure sucrose solutions-Continued

\begin{tabular}{|c|c|c|c|c|c|c|c|c|c|}
\hline $\begin{array}{c}\text { Brix } \\
\text { or true } \\
\text { per cent } \\
\text { d. s. by } \\
\text { weight }\end{array}$ & $\begin{array}{c}\text { True } \\
\text { density } \\
\text { (Plato) } \\
20^{\circ}\end{array}$ & $\begin{array}{l}\text { Appar- } \\
\text { ent den- } \\
\text { sity } 20^{\circ}\end{array}$ & $\begin{array}{l}\text { Appar- } \\
\text { ent spe- } \\
\text { cific } \\
\text { gravity } \\
20^{\circ} / 20^{\circ}\end{array}$ & $\begin{array}{l}\text { g sucrose } \\
\text { per } 100 \mathrm{ml} \\
\text { weight } \\
\text { in vacuo }\end{array}$ & $\begin{array}{l}\text { Brix } \\
\text { or true } \\
\text { per cent } \\
\text { d. s. by } \\
\text { weight }\end{array}$ & $\begin{array}{c}\text { True } \\
\text { density } \\
\text { (Plato) } \\
20^{\circ}\end{array}$ & $\begin{array}{l}\text { Appar- } \\
\text { ent den- } \\
\text { sity } 20^{\circ}\end{array}$ & $\begin{array}{l}\text { Appar- } \\
\text { ent spe- } \\
\text { cific } \\
\text { gravity } \\
20^{\circ} / 20^{\circ}\end{array}$ & $\begin{array}{l}\text { g sucrose } \\
\text { per } 100 \mathrm{ml} \\
\text { weight } \\
\text { in vacuo }\end{array}$ \\
\hline 1 & 2 & 3 & 4 & 5 & 1 & 2 & 3 & 4 & 5 \\
\hline $\begin{array}{l}82.0 \\
82.1 \\
82.2 \\
82.3 \\
82.4\end{array}$ & $\begin{array}{r}1.42507 \\
574 \\
642 \\
709 \\
776\end{array}$ & $\begin{array}{r}1.42407 \\
475 \\
543 \\
610 \\
677\end{array}$ & $\begin{array}{r}1.42810 \\
878 \\
946 \\
1.43013 \\
080\end{array}$ & $\begin{array}{r}116.856 \\
117.053 \\
.252 \\
.449 \\
.647\end{array}$ & $\begin{array}{l}83.0 \\
83.1 \\
83.2 \\
83.3 \\
83.4\end{array}$ & $\begin{array}{r}1.43181 \\
248 \\
316 \\
383 \\
451\end{array}$ & $\begin{array}{r}1.43081 \\
148 \\
216 \\
283 \\
351\end{array}$ & $\begin{array}{r}1.43486 \\
553 \\
621 \\
688 \\
756\end{array}$ & $\begin{array}{r}118.840 \\
119.039 \\
.239 \\
.438 \\
.638\end{array}$ \\
\hline $\begin{array}{l}82.5 \\
82.6 \\
82.7 \\
82.8 \\
82.9\end{array}$ & $\begin{array}{r}843 \\
911 \\
978 \\
1.43046 \\
113\end{array}$ & $\begin{array}{r}744 \\
811 \\
878 \\
946 \\
1.43013\end{array}$ & $\begin{array}{l}148 \\
214 \\
282 \\
350 \\
417\end{array}$ & $\begin{array}{r}.845 \\
118.044 \\
.243 \\
.442 \\
.641\end{array}$ & $\begin{array}{l}83.5 \\
83.6 \\
83.7 \\
83.8 \\
83.9\end{array}$ & $\begin{array}{l}519 \\
587 \\
654 \\
722 \\
790\end{array}$ & $\begin{array}{l}419 \\
488 \\
555 \\
623 \\
691\end{array}$ & $\begin{array}{r}824 \\
894 \\
961 \\
1.44029 \\
097\end{array}$ & $\begin{array}{r}.838 \\
120.039 \\
.238 \\
.439 \\
.640\end{array}$ \\
\hline
\end{tabular}

Washington, December 19, 1932. 